\title{
Choosing sides in the battle against gastric acid
}

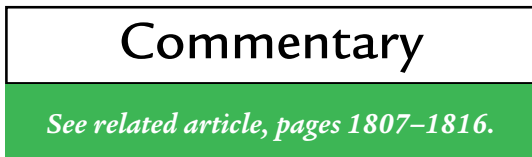

\author{
Marshall H. Montrose \\ Department of Cellular and Integrative Physiology, Indiana University, \\ 635 Barnhill Drive, Indianapolis, Indiana 46236, USA. \\ Phone: (317) 278-3674; Fax: (317) 278-3840; E-mail: mmontros@iupui.edu.
}

J. Clin. Invest. 108:1743-1744 (2001). DOI:10.1172/JCI200114652.

The duodenum is the first segment of the small intestine, just distal to the gastric pylorus. This positions the epithelial lining of the duodenum directly in the path of destructive gastric acid leaving the stomach. Because pancreatic bicarbonate secretions only enter the gut lumen farther down the gastrointestinal tract, the early duodenum must defend itself, unaided, from this acidic stress. The duodenal defenses can be conveniently grouped as pre-epithelial (e.g., neutralization of acid in the lumen), epithelial (e.g., tight junctions, membrane impermeability to acid, secretion of alkali and mucus), and subepithelial (e.g., afferent and efferent neural mechanisms, blood flow). Each of these contributions to mucosal defense is potentially important to human health because acid damage is a crucial aggravating factor in formation and persistence of duodenal ulcers (1).

In terms of duodenal defense, strong interest has centered on the avid bicarbonate secretion mediated by duodenal epithelial cells. This is unequivocally the first line of defense, as the bicarbonate reaching the gut lumen will act to neutralize intraluminal acid (producing $\mathrm{CO}_{2}$ and water) before it ever reaches the cells. Elegant and rigorous experiments have shown that, as a consequence of this neutralization, the spaces directly adjacent to the epithelium have close to a neutral $\mathrm{pH}$ $(2,3)$. This juxtamucosal alkaline layer also contributes to mucosal defense by directly shielding duodenal epithelial cells from luminal acid. The article by Akiba et al. in this issue of the JCI (4) is interesting and important because it raises a new possibility: that bicarbonate secretory mechanisms may have an even greater role in acid defense through their effects on intracellular $\mathrm{pH}$ of duodenal epithelial cells.
The central observation reported by Akiba et al. is that two anion transport inhibitors (DIDS and NPPB) both block bicarbonate secretion into the lumen but that these agents have opposite effects on cellular damage in response to luminal acid (4). Most strikingly, NPPB actually reduces the damage in response to luminal acid. These results are not readily compatible with conventional models of extracellular defense, since inhibition of bicarbonate secretion will compromise both luminal neutralization of acid and the juxtamucosal alkaline layer. Despite uncertainty about the molecular specificity of the drugs, these new in vivo results clearly demonstrate a dissociation between bicarbonate secretion and cytoprotection.

\section{Is intracellular $\mathrm{pH}$ more important than extracellular $\mathrm{pH}$ ?}

The authors extend their findings by measuring cytosolic $\mathrm{pH}$ in duodenal epithelial cells and showing that DIDS causes cellular acidification, whereas NPPB causes cellular alkalinization (4). They hypothesize that, at least in their specific condition, cytoprotection is mediated by bicarbonate transport, creating a relatively alkaline $\mathrm{pH}$ on the inside rather than the outside of the epithelial cell. If this hypothesis is correct, a corollary is that such effects on intracellular $\mathrm{pH}$ would be the dominant protective effect compared with extracellular $\mathrm{pH}$ changes. Neither hypothesis can be proven yet, because the mechanisms of DIDS and NPPB action remain speculative, and it is unknown whether changes in extracellular $\mathrm{pH}$ occurred as predicted in these experiments. The authors speculate that NPPB inhibits bicarbonate efflux from the cell via the apical cystic fibrosis transmembrane conductance regulator (CFTR) anion channel, leading to an accumulation of intracellular bicarbonate and consequent alkalinization (4). In contrast, DIDS might inhibit basolateral bicarbonate uptake via $\mathrm{NBC} 1$-mediated $\mathrm{Na}^{+} / \mathrm{HCO}_{3}{ }^{-}$cotransport, causing cellular acidification in the absence of adequate alkali uptake. The ideas are readily compatible with current evidence about bicarbonate transport routes, although molecular mechanisms of duodenal bicarbonate secretion remain controversial (5).

The putative site of NPPB action is particularly intriguing, since CFTR has been commanding attention as an essential component of duodenal bicarbonate secretion. Duodenal bicarbonate secretion is absent in CFTR knockout mice lacking functional CFTR (6-8). The simplest interpretation is that CFTR acts as a bicarbonate-conducting anion channel, although it could also be an essential regulator of another bicarbonate efflux route (9, 10). With respect to this scenario, Akiba et al. (4) highlight the potential relevance of the cytoprotective effect of NPPB by citing published clinical observations that cystic fibrosis (CF) patients (also lacking a functional CFTR protein) have fewer peptic ulcers than do normal individuals. This is especially intriguing since CF patients have normal gastric acid secretion, but they lack both duodenal and pancreatic alkali secretion. While a very attractive parallelism, the depth of the comparison remains to be proven. The basal cytosolic $\mathrm{pH}$ levels of isolated (unpolarized) duodenal enterocytes from normal and CF patients have been similar, although their $\mathrm{pH}$ regulatory mechanisms do differ (11). Further, there is significantly more peptic ulcer disease in African-American CF patients than in Caucasian CF patients, suggesting that genotype/phenotype correlations 
may need to be examined (12). However, Akiba et al. (4) should be credited with giving the first evidence to suggest a potential (and testable) mechanism for a puzzling clinical observation.

\section{Where in the duodenum is bicarbonate secreted?}

One controversial feature of the paper is that the experiments are designed to study $\mathrm{pH}_{\mathrm{i}}$ and damage at the villus tip. Conventional wisdom holds that bicarbonate secretion is a function of Brunner's glands, duodenal crypt glands distant from villi. Hence, the cells physiologically mediating bicarbonate secretion may not be the same as those examined to assess cytoprotection. However, there is no compelling evidence that bicarbonate secretion is restricted (or even localized) to Brunner's glands, structures that may play a greater role in secretion of mucin and bioactive peptides (13). Most relevant to this study, initial in situ hybridization studies localized CFTR mRNA exclusively to crypts in human duodenum (14). However, CFTR protein has been observed all along the villus/crypt axis in human and mouse duodenum, albeit with greater staining in crypts (15-17). In rats, evidence suggests the presence of an infrequent, highly CFTR-expressing cell interspersed among villus cells (18). Ultimately, where bicarbonate is secreted may not matter as much as where it provides protection. The work of Akiba et al. (4) makes a compelling case that blocking bicarbonate secretion in vivo can increase or decrease acid damage to cells at the villus tip.

Overall, the article by Akiba et al. (4) shifts attention about benefits of bicarbonate secretion from the outside of the cell to the inside of the cell. There are now some fascinating and pressing questions about the response of duodenal cells in CFTR knockout mice to acidic stress. In that model system, there will also be a unique opportunity to evaluate whether NPPB is having cytoprotective effects other than via direct effects on CFTR. Thus, while Akiba et al. (4) successfully drive a wedge between the long-standing correlations of enhanced bicarbonate secretion and enhanced mucosal protection, it remains to be seen whether that wedge can be driven deeper.

1. Allen, A., Flemstrom, G., Garner, A., and Kivilaakso, E. 1993. Gastroduodenal mucosal protection. Physiol. Rev. 73:823-857.

2. Paimela, H., Kiviluoto, T., Mustonen, H., and Kivilaakso, E. 1992. Intracellular $\mathrm{pH}$ in isolated Necturus duodenal mucosa exposed to luminal acid. Gastroenterology. 102:862-867.

3. Flemstrom, G., and Kivilaakso, E. 1983. Demonstration of a $\mathrm{pH}$ gradient at the luminal surface of rat duodenum in vivo and its dependence on mucosal alkaline secretion. Gastroenterology. 84:787-794

4. Akiba, Y., et al. 2001. Cellular bicarbonate protects rat duodenal mucosa from acid-induced injury. J. Clin. Invest. 108:1807-1816.

5. Flemstrom, G., and Isenberg, J.I. 2001. Gastroduodenal mucosal alkaline secretion and mucosal protection. News Physiol. Sci. 16:23-28.
6. Seidler, U., et al. 1997. A functional CFTR protein is required for mouse intestinal cAMP-, cGMPand $\mathrm{Ca}(2+)$-dependent $\mathrm{HCO} 3$ - secretion. J. Physiol. 505:411-423.

7. Hogan, D.L., et al. 1997. Acid-stimulated duodenal bicarbonate secretion involves a CFTR-mediated transport pathway in mice. Gastroenterology. 113:533-541.

8. Hogan, D.L., et al. 1997. CFTR mediates cAMPand $\mathrm{Ca} 2+$-activated duodenal epithelial $\mathrm{HCO} 3$ secretion. Am. J. Physiol. 272:G872-G878.

9. Clarke, L.L., and Harline, M.C. 1998. Dual role of CFTR in cAMP-stimulated $\mathrm{HCO} 3$ - secretion across murine duodenum. Am. J. Physiol. 274:G718-G726.

10. Poulsen, J.H., Fischer, H., Illek, B., and Machen, T.E. 1994. Bicarbonate conductance and $\mathrm{pH}$ regulatory capability of cystic fibrosis transmem brane conductance regulator. Proc. Natl. Acad. Sci. USA. 91:5340-5344.

11. Pratha, V.S., et al. 2000. Identification of transport abnormalities in duodenal mucosa and duodenal enterocytes from patients with cystic fibrosis. Gastroenterology. 118:1051-1060.

12. McColley, S.A., Rosenstein, B.J., and Cutting, G.R. 1991. Differences in expression of cystic fibrosis in blacks and whites. Am. J. Dis. Child. 145:94-97.

13. Krause, W.J. 2000. Brunner's glands: a structural, histochemical and pathological profile. Prog. His tochem. Cytochem. 35:259-367.

14. Strong, T.V., Boehm, K., and Collins, F.S. 1994 Localization of cystic fibrosis transmembrane conductance regulator mRNA in the human gastrointestinal tract by in situ hybridization. J. Clin. Invest. 93:347-354.

15. Kalin, N., Claass, A., Sommer, M., Puchelle, E., and Tummler, B. 1999. DeltaF508 CFTR protein expression in tissues from patients with cystic fibrosis. J. Clin. Invest. 103:1379-1389.

16. Hoogeveen, A.T., et al. 1991. Immunological localization of cystic fibrosis candidate gene products. Exp. Cell Res. 193:435-437.

17. Ameen, N., Alexis, J., and Salas, P. 2000. Cellular localization of the cystic fibrosis transmembrane conductance regulator in mouse intestinal tract. Histochem. Cell Biol. 114:69-75.

18. Ameen, N.A., et al. 1999. CFTR channel insertion to the apical surface in rat duodenal villus epithelial cells is upregulated by VIP in vivo. J. Cell Sci. 112:887-894. 